\title{
The Effectiveness of Visual Prompt on Clothing Recycling Behaviors
}

\author{
Yumeng He, Runian Wang* \\ Department of Marxism, China Pharmaceutical University, Nanjing, China \\ Email: *cpu2019@126.com
}

How to cite this paper: He, Y.M. and Wang, R.N. (2019) The Effectiveness of Visual Prompt on Clothing Recycling Behaviors. Open Journal of Social Sciences, 7, 390-395.

https://doi.org/10.4236/jss.2019.74031

Received: March 28, 2019

Accepted: April 26, 2019

Published: April 29, 2019

Copyright (C) 2019 by author(s) and Scientific Research Publishing Inc. This work is licensed under the Creative Commons Attribution International License (CC BY 4.0).

http://creativecommons.org/licenses/by/4.0/

(c) (i) Open Access

\begin{abstract}
This paper attempts to verify the effectiveness of visual prompt on clothing recycling behaviors by Randomised Control Trial (RCT) in a community in China, which covered 3000 households. During a twenty-week experiment (4 weeks for baseline, 4 weeks for experiment and four-week follow-up 8 weeks after experimental weeks), separately collected old clothing were counted (in piece), recorded and analyzed in both treatment $(\mathrm{N}=1500$ households within 15 buildings on the east side) and control groups ( $\mathrm{N}=1500$ households within 15 buildings on the west side). The self-designed visual prompt was pasted on the old clothing recycling bin in the treatment group area only. Results showed that, there was no significant change in the account of old clothing captured for recycling between the control group and treatment group during baseline period. However, there was a significant increase in the treatment group during experimental period, and individuals' clothing recycling behaviors persisted during the follow-up period, which means visual prompt could exert significant and sustainable effect on people's clothing recycling behaviors.
\end{abstract}

\section{Keywords}

Visual Prompt, Effectiveness, Clothing Recycling Behaviors

\section{Introduction}

According to the World Bank report, there are still 1.2 billion people in the world who are extremely poor, with more than 200 million people living below the poverty line in China. Old clothing can be distributed to the poor in remote areas to alleviate the problem of dressing, as well as be recycled for production reuse. Encouraging clothing recycling behaviors is an effective way to help the poor supplement the source of clothing, and is also necessary for building a 
conservation-oriented society and promoting sustainable development.

Visual prompt is actually a kind of visual stimulation, through which guides and persuades individuals to implement expected behaviors. It has been proven that visual prompt has the function of promoting pro-environmental behaviors, such as waste recycling [1] [2] [3] [4] [5]. Clothing recycling behavior was just as significantly meaningful as other recycling behaviors. But what surprised us was that few studies have focused on how to promote clothing recycling behaviors. Based on that, this paper tried to verify if visual prompt could promote individuals' clothing recycling behaviors significantly and persistently, which aimed to research and provide an effective way of encouraging people's recycling behaviors.

\section{Methods}

\subsection{Volunteers and Settings}

A community with 3000 households was studied in China, which was divided into two parts artificially and equally. 15 buildings on the east side were set as the treatment group, and the other 15 buildings on the west side were set as the control group. In order to improve crowd coverage, the old clothing recycling bins were located at the entrance of each side. And the visual prompt was pasted on the bin which was set near the east entrance only. Four but not two volunteers, who were all university freshman and the average age is 19 , were chosen to count and record the number of old clothing put into the recycling bin, in order to guarantee the continuity and integrity of data. Volunteers were required to check and record the number of clothing every day (including weekends) at about $10 \mathrm{pm}$ and transfer the clothing to a designated location if the recycling bin was full. Each volunteer checked the same recycling bin from the beginning to the end.

\subsection{Details of the Visual Prompt}

As was shown in Figure 1, the visual prompt used in this study was a sticker that consists of text, data and photo. In terms of text and data, the primary message was designed in large black bold capitals on a grey background, read: "DONATE YOUR OLD CLOTHING PLEASE". The secondary message included some data which was enlarged and in different colour, aiming to deepen people's understanding of the urgency and necessity of recycling old clothing by displaying data of world and China's poor population. "According to the World Bank report, there are still 1.2 billion people in the world who are extremely poor, with more than 200 million people living below the poverty line in China." At the bottom of the sticker, there was some information telling individuals where the clothing are going. "The clothing you donate will be transferred to the relevant charity and eventually reach the poor areas." The photo was set on the top of the sticker, aiming to attract people's attention to a greater extent. In short, the visual prompt was designed as attractive as possible to persuade individuals into recycling their old clothing. In the process of design, we consulted the teachers, classmates and some passers-by, and referred to some previous research on design aspects [6] 


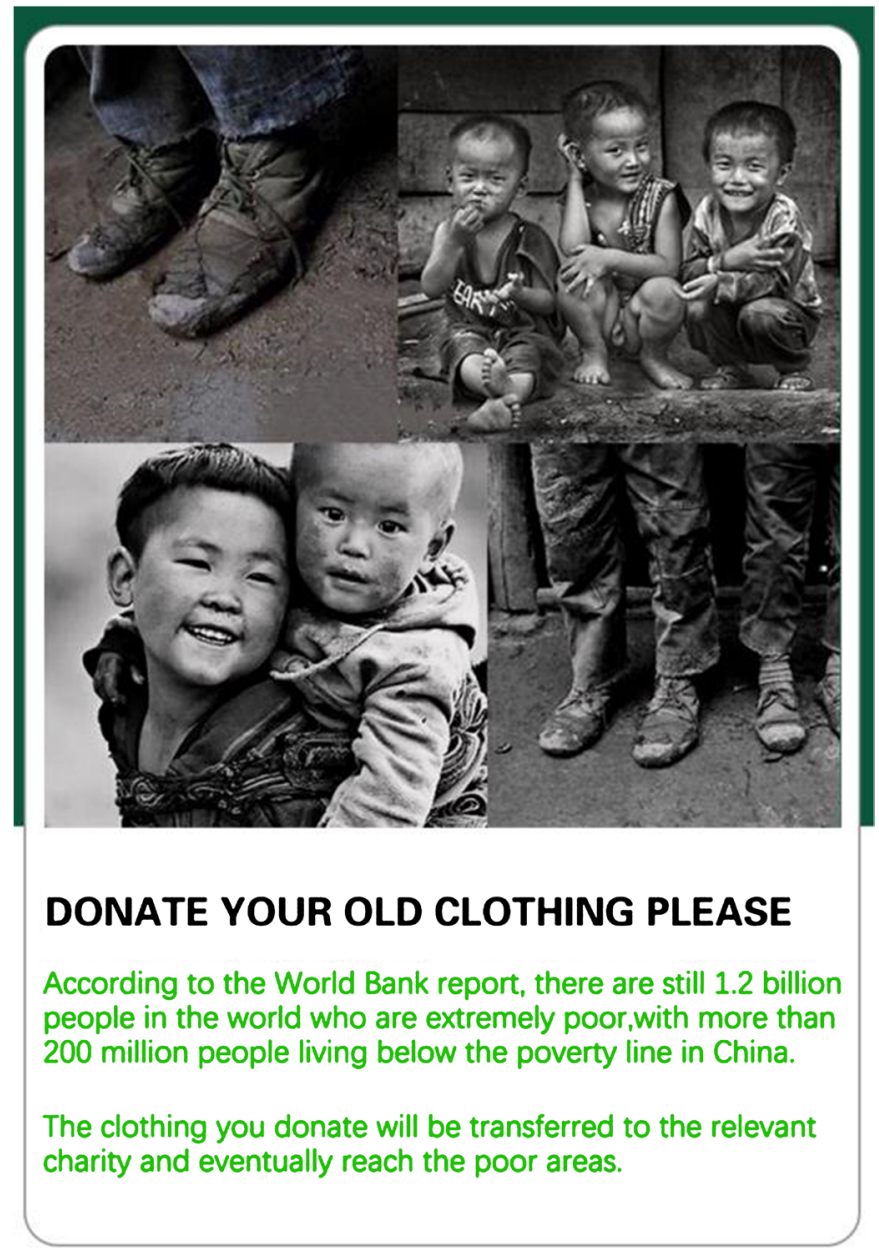

Figure 1. Sticker visual prompt.

[7] [8] [9] [10]. The visual prompt was A5 $(148 \times 210 \mathrm{~mm})$ in size in order to fit neatly onto the old clothing recycling bin.

\subsection{Procedures}

This experiment lasted for 20 weeks with an interval of 8 weeks. The former 4 weeks were the baseline of the experiment, aiming to get the background value of individuals' clothing recycling behaviors. During the next 4 weeks, the self-designed visual prompt was pasted on clothing recycling bin of the treatment group, and no change happened to the control group, so as to compare the difference between with or without visual prompt. The visual prompt was then reserved continuously and 8 weeks later, four-week follow-up was conducted to verify if visual prompt could exert sustainable effect on people's clothing recycling behaviors.

\section{Results}

\subsection{About the Visual Prompt}

Observing the status of the visual prompt was also a responsibility of the volun- 
teers. If the visual prompt was painted or damaged or fell off, the volunteer was asked to change a new one. Fortunately, none of the above phenomena occurred, which helped improve the accuracy and effectiveness of data collected.

\subsection{About Data of Clothing for Recycling}

The data collected by the volunteers was imported into spss 19.0 for data processing. As was shown in Table 1, the average of clothing for recycling of the control group was $1.75 \pm 1.26$ piece, when was $2.50 \pm 2.38$ piece of the treatment group. In terms of the amount of old clothing captured for recycling, there was no significant difference between control group and treatment group during the baseline period $(\mathrm{P}>0.05)$. But as was shown in Table 2, the average of clothing for recycling of the treatment group increased to $9.25 \pm 2.22$ piece, when only $3.75 \pm 2.99$ piece of the control group. $\mathrm{P}=0.025$ indicated that there was significant difference between control group and treatment group during the experimental period $(\mathrm{P}<0.05)$, Which means self-designed visual prompt had significant influence on promoting individuals' recycling behaviors. Statistical analysis in Table 3 indicated that the average of clothing for recycling of the control group and the treatment group was numerical proximity and according to $P$, there was no difference between experimental and follow-up period of treatment group $(\mathrm{P}>0.05)$, meaning that individuals' recycling behaviors persisted during the follow-up period and it is an effective way to promote individuals' clothing recycling behaviors by presenting visual prompt.

\section{Discussion}

There are several potential explanations for the impact of this intervention. Firstly, visual prompt in this study was designed as the form of sticker, and was

Table 1. Clothing captured for recycling in baseline period.

\begin{tabular}{ccc}
\hline Group & Mean \pm SD & P \\
\hline Control group & $1.75 \pm 1.26$ & 0.598 \\
Treatment group & $2.50 \pm 2.38$ & \\
\hline
\end{tabular}

Table 2. Clothing captured for recycling in experimental period.

\begin{tabular}{ccc}
\hline Group & Mean \pm SD & P \\
\hline Control group & $3.75 \pm 2.99$ & 0.025 \\
Treatment group & $9.25 \pm 2.22$ & \\
\hline
\end{tabular}

Table 3. Clothing for recycling of treatment group during experimental and follow-up period.

\begin{tabular}{ccc}
\hline Group & Mean \pm SD & P \\
\hline Control group & $9.25 \pm 2.22$ & 0.830 \\
Treatment group & $9.75 \pm 3.86$ & \\
\hline
\end{tabular}


pasted firmly to the lids of clothing recycling bin, which achieved longer-term exposure compared with leaflets or posters that might only be read once before they are discarded., increasing the opportunities for them to be noticed and the message to be conveyed. Secondly, realistic pictures and related data were added in the visual prompt, which make people understand the meaning of recycling clothing and greatly stimulate people's desire to donate clothes. Thirdly, individuals always discard old clothing with garbage under the condition without recycling bin. For such individuals, the prompt may serve as a persistent reminder that they should be separating out their old clothing. It seems that sticker visual prompt could be an effective medium for capturing the attention of a target audience, as they allow for information to be processed sufficiently [11].

While the research results are in line with expectations, there were also several limitations. Firstly, about limited research cycle. This study aimed to verify the effectiveness of visual prompt on clothing recycling, so as to provide an effective as well as economical way for promoting pro-environmental behavior. Based on that, longer time intervention and monitoring was necessary to examine if and when effects begin to "drop-off" and to what extent. Without monitoring the effects that occur during the full term of the payback period, the authors cannot conclusively determine whether the intervention is in fact truly cost effective. Study of larger scale and longer cycle should be conducted in the future research. Secondly, about the limited design of visual prompt. Although the sticker was designed with many people's opinions, its visual impact was still not strong enough, which affected the intervention effectiveness to some degree. More factors and advice, especially feedback of the respondent should be taken account into the design of visual prompt to get better visuals. Finally, about the absence of manipulation check, resulting in fuzzy data of the residents who received a sticker actually noticed its presence. Short feed back should be added to evaluate residents' perceptions of the sticker in future study.

\section{Conclusions}

The aim of the present study was to investigate the effectiveness of visual prompt on clothing recycling behaviors. It was predicted that sticker prompt would effectively and significantly increase the amount of clothing for recycling in the treatment group, and people's recycling behaviors would persist in follow-up period, which was consistent with the actual research results. During the baseline period, there was no significant difference between the control group and the treatment group. But when the sticker was pasted to the clothing recycling bin, the amount of clothing for recycling of the treatment group increased significantly. Although with an interval of 8 weeks, the impact of visual prompt still maintained.

It was important that the intervention was affordable as well as effective. The total cost for printing and distributing the sticker prompt was just $15 \$$ per community in this study (one sticker prompt for one clothing recycling bin), not 
including the cost of sticker design or the cost of data analysis as these were both completed by the staff members of the research group. Therefore, it is an economical way to promote individuals' clothing recycling behaviors by visual sticker prompt, as well as practical to achieve scaled applications.

\section{Conflicts of Interest}

The authors declare no conflicts of interest regarding the publication of this paper.

\section{References}

[1] Shearer, L., Gatersleben, B., Morse, S., Smyth, M. and Hunt, S. (2017) A Problem Unstuck? Evaluating the Effectiveness of Sticker Prompts for Encouraging Household Food Waste Recycling Behaviour. Waste Management, 60, 164-172. https://doi.org/10.1016/j.wasman.2016.09.036

[2] Lin, Z.Y., Wang, X., Li, C.J., Gordon, M.P.R. and Harder, M.K. (2016) Visual Prompts or Volunteer Models: An Experiment in Recycling. Sustainability, 8, 458. https://doi.org/10.3390/su8050458

[3] Miller, N.D., Meindl, J.N. and Caradine, M. (2016) The Effects of Bin Proximity and Visual Prompts on Recycling in a University Building. Behavior and Social Issues, 25, 4. https://doi.org/10.5210/bsi.v25i0.6141

[4] Clayton, M.C. and Blaskewicz, J. (2012) The Use of Visual Prompts to Increase the Cleanliness of Restrooms on a College Campus. Journal of Organizational Behavior Management, 32, 329. https://doi.org/10.1080/01608061.2012.729393

[5] Feng, Z.L. and Zhang, F.Z. (2019) Can Light-Shaped Visual Prompt Promote Individuals' Lights Off Behaviors More Effectively Than Ordinary One? Psychology, 10, 79-87. https://doi.org/10.4236/psych.2019.101006

[6] Aronson, E. and O'Leary, M. (1983) The Relative Effectiveness of Models and Prompts on Energy Conservation: A Field Experiment in a Shower Room. Journal of Environmental Systems, 12, 219-224. https://doi.org/10.2190/ubd5-4y9b-61ef-wum6

[7] Jae, H., Delvecchio, D. and Cowles, D. (2008) Picture-Text Incongruency in Print Advertisements among Low- and High-Literacy Consumers. Journal of Consumer Affairs, 42, 439-451. https://doi.org/10.1111/j.1745-6606.2008.00117.x

[8] Roberts, N., Mohamed, Z., Wong, P., Johnson, M., Loh, L. and Partridge, M. (2009) The Development and Comprehensibility of a Pictorial Asthma Action Plan. Patient Education and Counseling, 74, 12-18. https://doi.org/10.1016/j.pec.2008.07.049

[9] Sussman, R. and Gifford, R. (2012) Please Turn Off the Lights: The Effectiveness of Visual Prompts. Applied Ergonomics, 43, 596-603. https://doi.org/10.1016/j.apergo.2011.09.008

[10] van Meurs, L. and Aristoff, M. (2009) Split-Second Recognition: What Makes Outdoor Advertising Work? Journal of Advertising Research, 49, 82-92. https://doi.org/10.2501/s0021849909090011

[11] Bernstad, A. (2014). Household Food Waste Separation Behavior and the Importance of Convenience. Waste Management, 34, 1317-1323. https://doi.org/10.1016/j.wasman.2014.03.013 